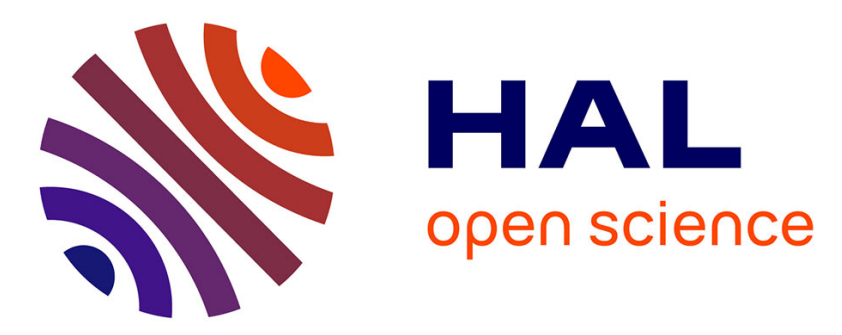

\title{
Differential Privacy in Tripartite Interaction: A Case Study with Linguistic Minorities in Canada
}

Arnaud Casteigts, Marie-Hélène Chomienne, Louise Bouchard, Guy-Vincent Jourdan

\section{- To cite this version:}

Arnaud Casteigts, Marie-Hélène Chomienne, Louise Bouchard, Guy-Vincent Jourdan. Differential Privacy in Tripartite Interaction: A Case Study with Linguistic Minorities in Canada. 7th International Workshop on Data Privacy Management (DPM), Sep 2012, Italy. pp.75-88. hal-00854281

\section{HAL Id: hal-00854281 https://hal.science/hal-00854281}

Submitted on 27 Aug 2013

HAL is a multi-disciplinary open access archive for the deposit and dissemination of scientific research documents, whether they are published or not. The documents may come from teaching and research institutions in France or abroad, or from public or private research centers.
L'archive ouverte pluridisciplinaire HAL, est destinée au dépôt et à la diffusion de documents scientifiques de niveau recherche, publiés ou non, émanant des établissements d'enseignement et de recherche français ou étrangers, des laboratoires publics ou privés. 


\title{
Differential Privacy in Tripartite Interaction: A Case Study with Linguistic Minorities in Canada
}

\author{
Arnaud Casteigts ${ }^{1,3}$, Marie-Hélène Chomienne ${ }^{1}$, Louise Bouchard ${ }^{2}$, \\ and Guy-Vincent Jourdan ${ }^{3}$ \\ 1 Institut de Recherche de l'Hôpital Montfort, Ottawa \\ ${ }^{2}$ Institute of Population Health, University of Ottawa \\ 3 School of Electrical Engineering and Computer Science, University of Ottawa
}

\begin{abstract}
This paper relates our venture to solve a real-world problem about official language minorities in Canada. The goal was to enable a form of linkage between health data (hosted at ICES - a provincial agency) and language data from the 2006 census (hosted at Statistics Canada - a federal agency) despite a seemingly impossible set of legal constraints. The long-term goal for health researchers is to understand health data according to the linguistic variable, shown to be a health determinant. We first suggested a pattern of tripartite interaction that, by design, prevents collection of residual information by a potential adversary. The suggestion was quickly set aside by Statistics Canada based on the risk of collusion an adversary could exploit among these entities. Our second suggestion was more involved; it consisted in adapting differential privacy mechanisms to the tripartite scheme so as to control the level of leakage in case of collusion. While not being rejected and even receiving enthousiastic interest per se, the solution was considered an option only if other simpler (but also less promising) alternatives are first, and methodically ruled out.
\end{abstract}

\section{Introduction}

\subsection{The Context}

Research in population health consists in studying the impact of various factors (determinants) on health, with the long-term objective of yielding better policies, programs, and services. Determinants of health are many, and researchers of Official Language Minority Communities (OLMCs) focus specifically on those related to speaking an official language in a minority context, such as English in Quebec, or French in the rest of Canada. (The setting for our study is the case of Francophone minorities in Ontario where English is the predominant official language.) Investigations of this type require, at the very minimum, the possibility of associating health data to linguistic information, whether at an individual or community level. Unfortunately, the largest health databases in Ontario, held at the Institute for Clinical Evaluative Sciences (ICES), do not include a linguistic variable to date. 
High-quality language variables from the 2006 Census exist at Statistics Canada (SC). Linking to the Census variables in the context of a punctual study is possible, and has already been done in Manitoba [1], but every such operation needs to satisfy a prescribed review and approval process [2]; it must also take place over a limited time, and requires to move the external data to be linked (health data, in our case) from its original source to $\mathrm{SC}$, which may not be achievable repeatedly.

We are interested in finding ways to exploit linguistic data from the 2006 Census on a regular and automated basis, by enabling its linkage to ICES health data in a dynamic way. It must be clear that we do not consider here a traditional type of linkage, in which data from both parts are matched to produce a somewhat larger amount of information of an individual nature (whether nominatively or anonymously). The linkage we consider is intrinsically transient and aggregated: it consists in allowing ICES to learn interactively how many Francophones are present in a given sample of individuals. This simple operation, referred to as a count query, can reveal a powerful building block to answer more complex questions about OLMCs, as we will see. Count queries are actually a particular type of tabulation, an operation already practiced at $\mathrm{SC}$ but that requires a manual process of verification. Turning it into something automated poses a number of challenges that we address here. A preliminary version of this paper also appeared in a technical report by the same authors [3].

We are concerned with ensuring privacy for both health and linguistic data. We suggested two possible mechanisms to enable dynamic count queries. The first consisted in a circular workflow between the three involved entities: health researchers, ICES, and SC. The workflow is initiated by the researcher through the submission of health criteria to ICES. A representative sample of individuals matching these criteria is then generated and sent to SC, which performs the count query. The result of the query is finally returned to the researcher. The privacy in this mechanism comes from the fact that the researcher does not know the sample details, SC does not know the health criteria that were used to generate that sample, and ICES does not know the final answer. This however assumes that no additional exchange of information occurs between the entities (no collusion). In particular, the assumption that ICES and the health researchers do not collude was rejected by SC, which considers by policy anything external as one and a single entity.

The second solution we proposed strives to prevent colluded attacks by means of adding noise to the queries answers. We found basic techniques in early literature on differential privacy [4, 5, 6, 6, , 8, 9, 10, 11] that fitted well our needs (more recent works have been done, but these seminal papers essentially contained all we needed). Differential privacy is concerned with understanding the precise impact of adding noise in privacy data analysis. We adapted these results to characterize what leakage is precisely at play in our scenario, and what parameters are involved in the tradeoff between leakage and utility. These results allowed us to make the point that using census data was technically feasible, which was well received by the SC establishment (and the subject of a talk we gave at their annual event: the Health Data Users Conference 2011). Of 
particular interest to them was the genericity of the approach and its potential to enrich provincial health databases more systematically with the range of federally available census data. After a short preamble introducing the main assumptions and basic problem, this paper describes both solutions and their applicability.

\section{$1.2 \quad$ Assumptions}

For the sake of clarity, we will make three assumptions. These assumptions will be used recurrently in the document, and relaxed (or discussed) in Section 4. They are:

1. The language of an individual can be fully described by a Boolean value $\{$ Franco, Anglo\}. (This over-simplistic view is erroneous, since language is a multi-dimensional variable.)

2. The census data comprises this variable relative to each individual. (In fact, the unit of census is the household, not the individual.)

3. It is possible to generate, from the ICES database, a representative sample of individuals matching a given set of health criteria. (From our discussions with ICES representatives, this seems to be a fair assumption.)

\subsection{Count Queries}

Given a sample of individuals $s$, and a property $p$, a count query $q(s, p)$ consists of counting the number of individuals in $s$ that satisfy $p$. We consider the basic count query illustrated on Figure 1 .

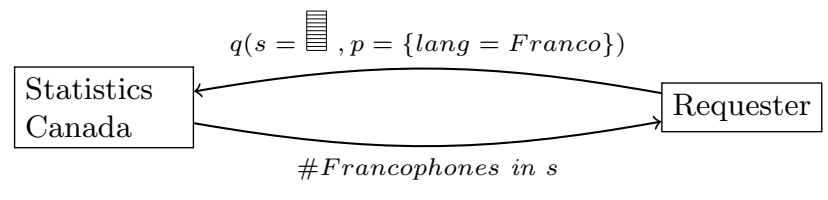

Fig. 1. A basic count query

As such, a sample of individuals is sent to SC, and SC answers back with the number of Francophones in that sample. To illustrate the potential of this simple query, let us consider the following questions:

1. In Ontario, what is the average angioplasty rate among Francophones? (vs. Anglophones)

2. In Ontario, what is the hospital utilization rate for $65+$ seniors with type- 2 diabetes? (Francophones vs. Anglophones)

As far as language is concerned, the use of count queries can answer these questions. In the first case, ICES would generate a representative sample of individuals having undergone angioplasty, then ask SC for a count query in that sample. By normalizing the resulting answer over the more general ratio of Francophones 
in Ontario (which is known), one can answer the initial question. We can apply a variation of this method to the second example, by using several samples in various ranges of utilization rates by $65+$ diabetic patients. Hence, a single mechanism (count query) on a single variable (language) seems sufficient - as far as SC is concerned - to answer a variety of OLMC-related questions.

The problem: A malicious use of count queries, if unsupervised, could make it possible to identify the language of a given individual (say, Madame $x$ ). Consider the following attack: making a query with a sample $s_{1}$ that does not contain $x$; then making a second query with the same sample, plus $x$ (see Figure 2). Obviously, if answer $\left(s_{2}\right)>$ answer $\left(s_{1}\right)$, then Madame $x$ is Francophone.

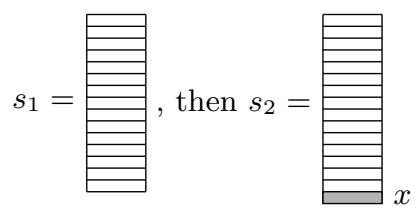

Fig. 2. Identifying the language of an individual by successive queries (basic example)

Even though language is supposedly not as 'sensitive' as other classes of information (e.g. health or income), SC's policy in regard of residual information is absolutely strict. Solutions to this problem are not trivial, even though one may think it suffices to prevent this particular scenario. More complex adversarial strategies could actually be designed. Our first solution avoids the problem by means of dataflow itself; while the second acknowledges the risk and characterizes what tradeoff is at play between leakage and utility with the addition of noise.

\section{First Mechanism: Tripartite Interaction}

The first (and simplest) mechanism aims at providing guaranties at the data workflow level. It consists of a tripartite interaction between researchers, ICES, and SC; each entity obtaining and contributing only the minimal amount of information required to carry out the count query. The workflow is depicted on Figure 3, and can be summarized in three chronological steps:

1. Researchers issue a set of criteria related to a research question (e.g., angioplasty rates, or hospital utilization rate in a $65+$ type 2 diabetes population);

2. ICES generates a sample of individuals responding to the criteria and sends it directly to $\mathrm{SC}$;

3. SC executes the language query on the generated sample, and answers directly to the researchers. 


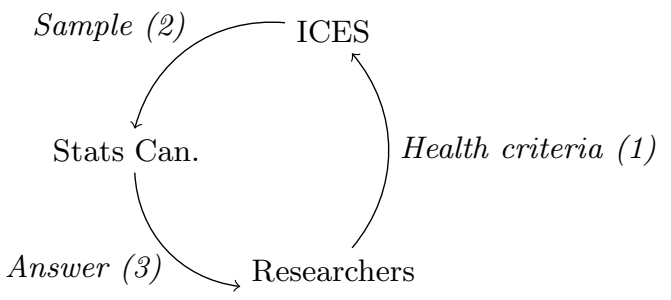

Fig. 3. Privacy by means of tripartite interaction

This scheme protects privacy by combining the three following facts:

1. SC does not know what health criteria the sample is associated with;

2. Health researchers do not know what sample is generated;

3. ICES does not know the result of the language query.

Privacy of ICES health data results from statements 1 and 2, whose combination implies that ICES remains at all times the only entity capable of associating individuals with the queried health criteria. Similarly, the combination of statements 2 and 3 implies that SC remains the only entity capable of associating a sample of individual with the number of Francophones within; such an association being necessary to collect residual information (and thus perform attacks like the one in Figure 2).

\section{Second Mechanism: Noisy Count Queries}

The first mechanism having been set aside by SC due to the possibility of identifying an individual's language if ICES and the health researchers collude as a single entity, we have proposed a more elaborate mechanism whereby random noise is added to the answers, as illustrated on Figure 4.

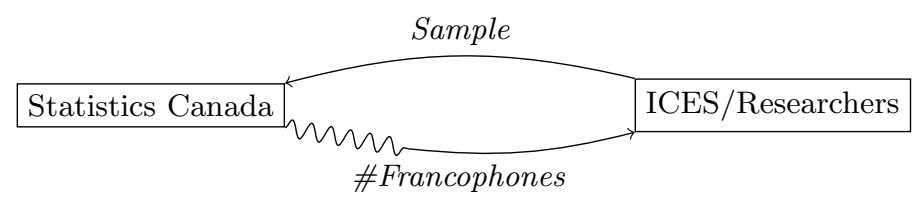

Fig. 4. Noisy count queries

As we will see in this section, this mechanism involves the interplay between three parameters: 1) the magnitude of the noise, 2) the level of statistical leakage tolerated by SC, and 3) the number of queries that can be performed (before shutting down the service). We have characterized this interplay mathematically, based on seminal results on differential privacy. 


\subsection{Overview of the Principle}

What makes possible an attack like that of Figure 2 on page 78 , and more generally the accumulation of residual information on individuals, is that the presence or absence of these individuals inside the sample does impact the answer. Suppose the exact answers for $s_{1}$ and $s_{2}$ are respectively 586 and 587 (i.e., Madame $x$ is Francophone) and consider adding or removing a small random number to the answer before returning it - we call it perturbing the answer with some noise. The resulting answers for $s_{1}$ and $s_{2}$ may be for example 589 and 584, or 583 and 587, or 585 and 585. Clearly, these answers do not leak Madame $x$ 's language, and still, they are meaningful to a researcher.

There exist different types of noise, the most common of which are binomial, Gaussian, or Laplacian noises (named after the distributions from which the random number is drawn). As we will see, Laplacian noise has good properties that makes it an appropriate choice in our case. We consider perturbing the answer by adding a random number drawn from a Laplace distribution (see the example on Figure 5). This distribution can be considered at various scales, depending on how sharp or flat the probabilities are concentrated around the true result.

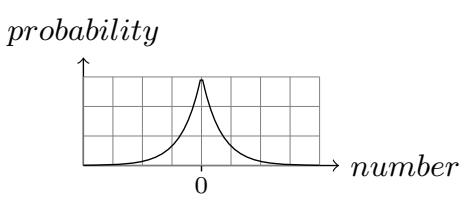

(a) Laplace distribution

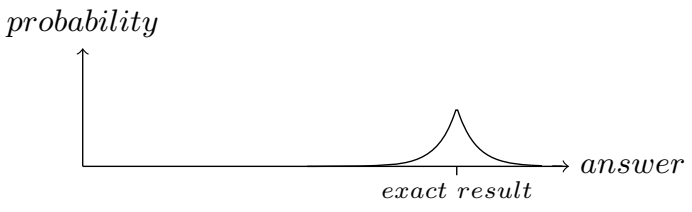

(b) Resulting probability of answer

Fig. 5. Addition of Laplacian noise

Intuitively, performing a small number of noisy queries will not leak much individual information, whereas repeating a similar operation many times could eventually leak something substantial. In fact, every query does leak a small amount of statistical information, the quantity of which depends on the noise magnitude (scale of the Laplace distribution).

Consider again the example with $s_{1}$ and $s_{2}$, and two different scenarios, where noise is added according to a small scale or a large scale, respectively. The resulting probabilities of answers are represented on Figure 6, In a worst-case scenario, assume the requester already knows the correct answer for $s_{1}$, and is querying the system with $s_{2}$. It eventually obtains a random answer, say $n$.

Figure 6 shows intuitively how the leakage depends on the noise scale. On the left, with an arbitrary small scale, answer $n$ is approximately 6 times more likely if Madame $x$ is Francophone. Thus, obtaining answer $n$ indirectly implies that Madame $x$ has $\sim 85.7 \%$ chances to be Francophone. With the larger scale, on the right, answer $n$ is about 1.3 more likely to occur if Madame $x$ is Francophone, 

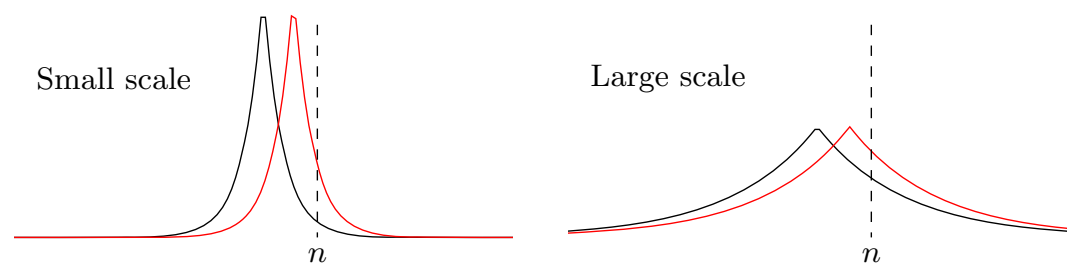

Fig. 6. Different magnitudes of noise. The black and red curves in each picture represent the probability of answer if Madame $x$ is non-Francophone or Francophone, respectively. The vertical bar represents a possible (and arbitrary) answer $n$.

inducing "only" a belief of $\sim 56.5 \%$ that she is Francophone. A convenient property of Laplacian noises is that the ratio between both curves does not depend on the exact position of $n$. If you choose $n$ further right, zoom in and measure again the ratio, it remains the same. The left side of the curves behaves symmetrically; as for the middle section between both peaks, it exhibits a varying ratio, but its value necessarily remains smaller than those on the right and left sides.

It is therefore possible to bound the amount of leakage such a query induces by choosing a desired noise magnitude. Now, one should keep in mind that while larger magnitudes of noise mean safer data, they also mean less accurate answers to the researchers. There is actually a tripartite tradeoff between noise, utility, and the number of queries that the system can hold.

\subsection{Detailed Principle}

Private data analysis research is at the confluence of cryptology, statistics, and database systems. It focuses on statistical exploitation of privately held data by means of dedicated mechanisms. Adding noise to a query is one of these mechanisms, referred to as output perturbation. This topic has seen a resurgence of interest in the past five years, mainly due to a conceptual shift in the definition of privacy.

Differential privacy: Access to a statistical database should not enable one to learn anything about an individual, given that its data is in [the tested sample], that could not be learnt otherwise.

This definition should be understood as follows. Consider that we know that 1) Madame $x$ underwent an angioplasty, and 2) $60 \%$ of angioplasty patients are Francophones (from a count query at SC). The subsequent belief that Madame $x$ has $60 \%$ chances of being a Francophone should not be considered as a leakage over Madame $x$ 's language, because the same belief could have been inferred with or without her being in the tested sample. (Such information is precisely what OLMC researchers attempt to learn.) 
Based on this definition, a new line of research was developed [4, [5, 6, 7, 8] around the question of how to limit the amount of information one can learn - leakage - about a specific individual that cannot be learned by means of an encompassing sample. The neighbor-sample setting discussed previously represents a worst-case scenario in this regard because the difference between both outputs can be attributed to a single individual; the leakage generated in this setting is therefore considered as an upper bound on the risk the database is more generally exposed to.

Relation between Noise and Leakage. Let us first imagine that only one query is performed over the whole lifetime of the system. (The extension to multiple queries is rather straightforward and discussed in a second step.) The main result from the field of differential privacy is to teach us how to generate the noise so as to observe a desired bound on the leakage, assuming this bound is expressed using the 'neighbor-sample' setting. Concretely, once this bound (let us note it param) is decided upon, differential privacy tells us how to generate the noise so as to ensure that

$$
\frac{P\left[\operatorname{answer}\left(s_{1}\right)=n\right]}{P\left[\operatorname{answer}\left(s_{2}\right)=n\right]} \leq \text { param }
$$

for any neighbor samples $s_{1}, s_{2}$ and any possible answer $n$. The scale of the noise is then determined based on param and on the sensitivity of the considered query; in our case, a count query. (The differential privacy framework is very general and applies to other types of queries; we will discuss some of them later in this document.)

Sensitivity: The sensitivity of a query, noted $\Delta q$, is defined as the maximal difference in output that two neighbor samples can induce. Two neighbor samples in our case cannot induce a difference larger than 1; thus, the sensitivity of count queries is 1 . Note that the sensitivity does not depend on the size of the sample. This may appear counter-intuitive, but is actually consistent with generating the noise irrespective of the sample size.

The formula: The main result from [8], subsequently simplified in [4], tells us that differential privacy is guaranteed with a given leakage bound 'param' if the noise added to each answer is generated according to a Laplace distribution of scale

$$
b=(\Delta q) / \log (\text { param })
$$

This is usually written Noise $\sim \operatorname{Lap}(0, b)$, where the first parameter simply indicates that the noise will be centered on the true result.

Number of queries: This formula composes very well in the case of a sequence of queries. Indeed, the above papers tell us that, in order to maintain the same level of leakage for a sequence of queries, it suffices to generate the noise as if that 
sequence was a single query whose sensitivity is the count of all the sensitivities. When all queries are of a same type, this comes to considering the scale

$$
b=(\# q \Delta q) / \log (\text { param })
$$

where $\# q$ is the number of queries in the sequence and $\Delta q$ is the sensitivity of every individual query. A convenient consequence is that the database holder can think of choosing 'param' irrespective of the number of queries.

Choosing the Leakage Parameter. A minimum of leakage is necessary for utility. If for instance param $=1$, which implies that the probability of answer must be exactly the same for any neighbor samples $s_{1}$ and $s_{2}$, the problem that arises is that any non-neighbor pair of samples can be indirectly connected through a chain of neighbor samples. By transitivity, choosing param $=1$ therefore implies that the sample has no impact on the answer, which is nonsense. How to choose param is context-specific, and generally not discussed in the above papers. We explore this question in the context of language queries at Statistics Canada.

Belief: From the point of view of SC, the easiest way to formulate an 'acceptable' level of leakage is to specify the maximal belief allowed on the language of an individual. Say, for example, that this limit is $80 \%$ (i.e., we should never believe that Madame $x$ has more than $80 \%$ chances to be Francophone or Anglophone).

Worst case: We are interested in determining what value of param should be chosen under the worst possible assumptions (mostly unrealistic). These assumptions are:

1. The adversary has no interest in health research; its only purpose is to learn Madame $x$ 's language.

2. The adversary has sufficient access in order to waste all the 'capital' of queries offered by SC in that sole purpose.

3. The adversary is able to build samples in which the language of each individual but Madame $x$ is known.

4. SC applies no filter on the queries.

Consider the same attack scenario as discussed in Section 3.1, based on two neighbor samples $s_{1}$ and $s_{2}$ (the second being equal to the first, plus Madame $x$ ). Because of point 3 , the adversary already knows the exact answer for $s_{1}$, and can therefore use all the credit of queries to play and replay $s_{2}$. Assume it eventually gets a final answer $n$. Whether $n$ corresponds to an average, a count, or any other combination of all the answers does not matter: differential privacy tells us that this answer cannot be param times more likely for $s_{2}$ than for $s_{1}$, which is the only thing that matters here.

Three cases are considered, depending on the position of $n$ with respect to the two probability peaks: if $n$ is on the right of the right peak, then Madame $x$ is param more likely to be Francophone than non-Francophone; and symmetrically, 
if $n$ is on the left of the left peak, she is param more likely to be Anglophone. In the middle section, she could be up to param more likely - but not more, as already discussed - to be either. Let us assume that $n$ is on the right of the right peak, while keeping in mind that the following reasonning applies symmetrically. This implies that

$$
\begin{aligned}
& \frac{P[x \text { is Francophone }]}{P[x \text { is not Francophone }]} \leq \text { param } \\
\Longrightarrow & \frac{P[x \text { is Francophone }]}{1-P[x \text { is Francophone }]} \leq \text { param } \\
& \ldots \\
\Longrightarrow P[x \text { is Francophone }] & \leq \text { param } /(1+\text { param })
\end{aligned}
$$

This formula can be used to decide what value of param corresponds to the maximal belief allowed by policy; in the case of our $80 \%$-example above, this corresponds to param $=4$. We now combine Equations 3 and 4 to represent the $3 \mathrm{D}$ tradeoff between maximal belief, noise scale, and number of queries allowed. A meaningful cut of this volume is shown on Figure $7(\mathrm{a})$

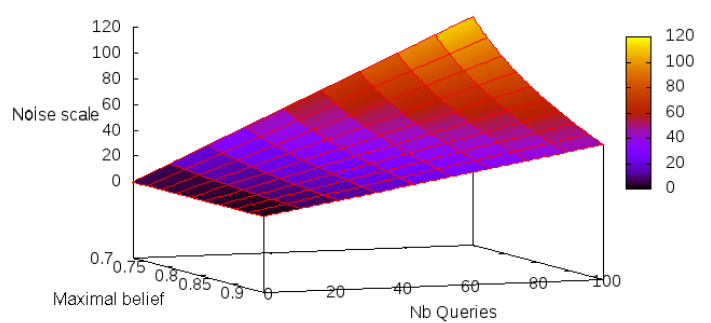

(a) Tradeoff between noise scale, number of queries, and maximal belief (multiply by 100 for percentage).

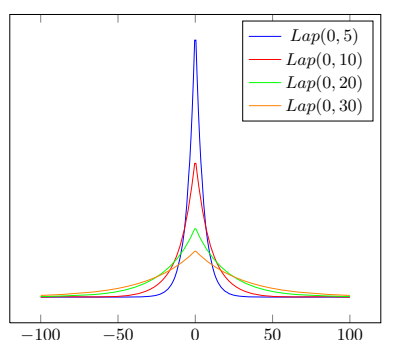

(b) Examples of noise scales.

Fig. 7. Choice of parameters

The right picture (Figure 7(b) represents several examples of noise scales. Essentially, it gives the intuition that the precision of an answer deteriorates quickly with the scale parameter. How this affects the quality of the results for a researcher depends on how large the samples are. If samples are large (say, larger than 10000), then a scale of 30, or even 50, may not be a problem. If, on the other hand, the samples sizes are in the order of 1000, then any scale larger than 20 or 30 will induce meaningless answers. (This also depends on the specific question investigated and how deep it involves the language determinant.)

To close this illustrative discussion, let us consider that researchers require a scale no larger than 30, and SC tolerates a maximal belief of $80 \%$, the tradeoff on Figure $7(\mathrm{a})$ tells us that up to $\sim 50$ queries are still possible. This is not a lot, but keep in mind that this tradeoff represents a totally unrealistic worst-case scenario, where a single adversary can (and decides to) play all the credit of queries on a single sample, in which the language of all individuals but Madame $x$ 's is already known. 


\subsection{Discussion}

What could be the consequences of relaxing some of the worst-case assumptions listed above? A first observation is that in our case, the researchers do not generate the samples directly; they do it through health criteria that are submitted to ICES (e.g. Figure 3), and the content of these samples is not known to the researchers. This implies that Assumption 3 is mostly unrealistic, and a given noisy answer will actually correspond to possibly more than two exact results. Further mathematical investigations are required to understand these implications precisely, but we can reasonably think that it would drastically lower the leakage that a query can possibly generate.

Another important aspect is to determine whether SC considers the couple ICES/researchers to be itself the adversary, or to be the potential victim of an external adversary. Put differently, does SC fear a direct misuse of the system, or a leak in data resulting from a normal-use? This point is crucial because the samples played in the context of a normal use are likely to generate much less leakage (intuitively, to the extent of a different order of magnitude).

As a third observation, Statistics Canada could easily filtrate the queries (Assumption 4) to prevent large intersection between the samples, and thereby drastically reduce the potential leakage of query combinations. Again, further mathematical investigation could be appropriate to explore, e.g., the correspondance between the size of intersection and the leakage. We believe that although difficult in the general case, this type of characterization remains reasonably feasible in the particular case of language-based count queries. We will contribute towards this direction if the option is concretely considered.

In regards to all these considerations, it is not senseless to believe that the number of possible count queries could actually jump to several thousands, while keeping the leakage below any reasonable level, e.g., much lower than a belief of $80 \%$. If unfortunately these somewhat optimistic observations are not considered by the involved players (desiring to stick to the worst-case model), then there will still be a way to satisfy all the parts. The solution would consist in deploying a non-bounded service, in which the cumulated leakage is progressively computed as the queries are performed, and the service is shutdown when the leakage has reached a given threshold.

\section{Relaxing the Three Initial Assumptions}

Language: We assumed heretofore that the language of an individual could be described by a boolean value $\{$ Franco, Anglo $\}$. Language is actually more subtle and requires looking at several aspects. In particular, the census data at SC includes the following variables: Mother tongue (for $100 \%$ of the population); Knowledge of official languages, Knowledge of non-official languages, First official language spoken, Language spoken at home, and Language of work (for $20 \%$ of the population). Also, some of these variables may include other options than French and English, as well as a possible combination of several languages. It is clear that a mere count query cannot precisely render the ratio of "Francophones" 
in a given sample; at the most it could for example count the number of persons who report French as mother tongue and not English (the census form allows to report several mother tongues). Fortunately, the differential privacy framework can easily be applied to more complex queries such as histograms, at the price of a slightly higher sensitivity per query [4].

Linkage technicalities: So far, we have assumed that a correspondence was technically feasible between an individual and its language variables at Statistics Canada. In fact, the unit of census is not the individual, but the household. Both the short-form and the long-form census comprise a nominative field, but this field is apparently optional and is known to contain, occasionally, some exotic answers like 'Mickey Mouse'. Three options are possible here: 1) Assume that the persons living in a same household share similar language profiles, and then build the query samples based on postal addresses; 2) Rely on the cleaning operation that is currently performed on the census data at Statistics Canada, and which recently allowed to reach the level of $15 \%$ of records linkable nominatively (all from the long-form subset); or 3) Set up a complementary linkage, housed at SC, between the census data and the minimal amount of administrative data allowing to associate insurance numbers with the corresponding census records. (Note that this represents only a subset of what was done for Manitoba as part of a larger linkage including health data directly at SC [1].)

Generation of the samples at ICES: Health information is highly-sensitive, and ICES has a strong policy of confidentiality in this regard (see [12]). Concretely, the database held at ICES is sanitized by replacing all nominative information by anonymous identifiers. Whether these identifiers can technically and lawfully be reversed to the original information is a question we are starting to investigate. It is likely that ICES maintains somewhere a private table with such associations. Thus, we believe that it is at least technically feasible. Such an operation should however be considered with utmost care. The feasibility also depends on what level of trust ICES grants to SC, even though SC does not need to know what health data is associated with a query (this is actually one of the advantages of the proposed mechanism). If need be, the body of research on cryptography techniques can be explored and leveraged. For instance, recent encryption methods have been proposed for the specific problem of querying databases using confidential inputs and criteria [13], that is in our case, preventing SC to learn what sample a given query is about.

\section{Concluding Remarks}

While not being rejected and even receiving enthousiastic interest per se, our second solution based on differential privacy was judged too difficult to deploy in the short term. Since other options exist that involve different (though less accurate) linguistic data, we were advised to explore these alternatives first and consider our solution only once the quality of these alternatives is shown insufficient for OLMC studies. In particular, efforts are being carried out at ICES to 
receive linguistic information from Ontario's Ministry Of Health And Long-Term Care (MOHLTC). Unfortunately, this information corresponds to the language individuals select for correspondence with the Ministry, which has potential for several biases and is not considered as reliable by OLMC researchers (in addition to being unidimensional). We are currently running an independent project to assess the quality of this variable, which, in case of negative results, will justify the deployment of stronger solutions like the one proposed here.

Acknowledgments. We are grateful to a number of persons that helped (and are still helping) us in this study. In particular, we want to thank Mariette Chartier, professor at the University of Manitoba, for her insightful comments and for sharing feedback on Manitoba experiments; Simone Dahrouge, scientist at C.T. Lamont primary health care center, for valuable information on ICES protocols and databases; Richard Trudeau, head of the health division at SC, for all the information on Statistics Canada rules and for mentioning the existence of tabulations; Michael Wolfson, formerly assistant chief statistician at SC (now professor at the University of Ottawa), for clarifying SC's point of view regarding the uniqueness of the ICES/researchers entity; Doug Manuel, Adjunct Scientist at ICES, for information on anonymization of health data; and Jean-Marie Berthelot, VP of Programs at the Canadian Institute for Health Information, for considerable insights about the diversity of parameters to take into account, and the possibly larger scope of this work. Finally, this study was made possible thanks to the financial support of the Ontarian Ministry of Health and Long-Term Care (through the AHRNI initiative and the RRASFO network), and the Institut de Recherche de l'Hôpital Montfort.

\section{References}

1. Houle, C., Berthelot, J.M., David, P., Wolfson, M.C., Mustard, C., Roos, L.: Matching census database and Manitoba health care files. In: Proc. of International Workshop on Record Linkage Techniques, p. 305. National Academies (1997)

2. Statistics Canada Website. Policy on record linkage (fetched September 2010), http://www.statcan.gc.ca/record-enregistrement/policy4-1-politique4-1eng.html

3. Casteigts, A., Chomienne, M.-H., Bouchard, L., Jourdan, G.-V.: Enabling Dynamic Linkage of Linguistic Census Data at Statistics Canada. Institut de Recherche de l'Hôpital Montfort, Technical report (March 2011)

4. Dwork, C., McSherry, F., Nissim, K., Smith, A.: Calibrating Noise to Sensitivity in Private Data Analysis. In: Halevi, S., Rabin, T. (eds.) TCC 2006. LNCS, vol. 3876, pp. 265-284. Springer, Heidelberg (2006)

5. Nissim, K., Raskhodnikova, S., Smith, A.: Smooth sensitivity and sampling in private data analysis. In: Proc. of 39th ACM Symposium on Theory of Computing (STOC), pp. 75-84 (2007)

6. Dwork, C.: Ask a Better Question, Get a Better Answer - A New Approach to Private Data Analysis. In: Schwentick, T., Suciu, D. (eds.) ICDT 2007. LNCS, vol. 4353, pp. 18-27. Springer, Heidelberg (2006) 
7. Dwork, C., Nissim, K.: Privacy-Preserving Datamining on Vertically Partitioned Databases. In: Franklin, M. (ed.) CRYPTO 2004. LNCS, vol. 3152, pp. 528-544. Springer, Heidelberg (2004)

8. Blum, A., Dwork, C., McSherry, F., Nissim, K.: Practical privacy: the SuLQ framework. In: Proc. of ACM Symposium on Principles of Database Systems (PODS), pp. $128-138(2005)$

9. Hardt, M., Talwar, K.: On the geometry of differential privacy. In: Proc. of the 42nd ACM Symposium on Theory of Computing (STOC), pp. 705-714 (2010)

10. Smith, A.: Privacy-preserving statistical estimation with optimal convergence rates. In: Proc. of the 43rd ACM Symposium on Theory of Computing (STOC), pp. 813-822 (2011)

11. Kifer, D., Machanavajjhala, A.: No free lunch in data privacy. In: Proc. of the 2011 Intl. Conf. on Management of Data, pp. 193-204 (2011)

12. Privacy at ICES (fetched March 2011), http://ices.queensu.ca/privacy.html

13. Stolfo, S.J., Tsudik, G. (eds.): IEEE Security \& Privacy Magazine, SI on PrivacyPreserving Sharing of Sensitive Information. IEEE Computer Society (July-August 2010) 